\title{
Periodic solutions for nonautonomous first order delay differential systems via Hamiltonian systems
}

\section{Qiong Meng*}

"Correspondence:

mengqiong@qq.com

School of Mathematical Science,

Shanxi University, Wucheng Road,

Taiyuan, Shanxi, China

\section{Springer}

\begin{abstract}
The existence of the nontrivial periodic solutions for the nonautonomous first order delay differential equation $x^{\prime}(t)=-[f(t, x(t-1))+f(t, x(t-2))+\cdots+f(t, x(t-(2 N-1)))]$ is investigated, where $f \in C(\mathbf{R} \times \mathbf{R}, \mathbf{R})$ is $2 N$-periodic in $t$ and odd in $x, N$ is a positive integer. We prove several new existence results by some recent critical point theorems.
\end{abstract}

MSC: 34K13; 34K18; 58E50

Keywords: first order delay differential equation; nonautonomous; periodic solution; critical point theorems

\section{Introduction}

It is well known that critical point theory is a powerful tool that deals with the multiplicity of periodic solutions to ordinary differential systems as well as partial differential equations (see [1-6]). In 1998, Li and He [7] firstly applied the critical point theory to study the multiplicity of periodic solutions to delay differential equations. Especially, in 2005, Guo and $\mathrm{Yu}[8]$ established a variational framework for autonomous systems. In the past several years, some results on the existence of periodic solutions for the functional differential equation were obtained by critical point theory (see [7-21]).

In 2006, Fei [11, 12] considered the following autonomous functional differential equation:

$$
(*) \quad x^{\prime}(t)=-[f(x(t-1))+f(x(t-2))+\cdots+f(x(t-(n-1)))],
$$

where $f$ is odd and $n \geq 2$ is an integer. Using the Yorke-Kaplan technique, $(*)$ is changed into a Hamiltonian system. Periodic solutions of the Hamiltonian systems are still obtained as critical points of a function $\varphi$ over a Hilbert space $E$. However, instead of finding critical points of $\varphi$ over $E$ directly, the author worked on a subspace of $E$ which has a symmetric structure. When $n$ is even, the function $\varphi$ is invariant and $\varphi$ is equivariant about a compact group. This allows one to find critical points of $\varphi$ on a subspace of $E$ which is invariant under the mentioned group action. Then one can apply the pseudo-index theory [2] to obtain periodic solutions in this subspace, which surely have the required symmetric structure and give solutions to $(*)$. When $n$ is odd, the function $\varphi$ is still invariant about a similar

(c) 2015 Meng; licensee Springer. This article is distributed under the terms of the Creative Commons Attribution 4.0 International License (http://creativecommons.org/licenses/by/4.0/), which permits unrestricted use, distribution, and reproduction in any medium, provided you give appropriate credit to the original author(s) and the source, provide a link to the Creative Commons license, and indicate if changes were made. 
compact group. However, $\varphi$ is not equivariant about this compact group action anymore. Therefore one cannot directly apply the same idea as in the case when $n$ is even. In order to overcome the difficulty, one has to construct equivariant pseudo-gradient vector fields and prove a new deformation theorem. Then one can combine a Galerkin approximation with the $S^{1}$-index theory ([4], Chapter 6) to obtain critical points of $\varphi$ with the required symmetric structure (for details see [12]).

But most of these functional differential equation are autonomous, and the results on the nonautonomous functional differential equation are relatively few (see $[9,15-19]$ ).

Motivated by the work of [10-12, 15-18], we consider a class of nonautonomous first order delay differential equations,

$$
x^{\prime}(t)=-[f(t, x(t-1))+f(t, x(t-2))+\cdots+f(t, x(t-(2 N-1)))]
$$

where $f \in C(\mathbf{R} \times \mathbf{R}, \mathbf{R})$ is $2 N$-periodic in $t$ and odd in $x, N \in \mathbf{Z}^{+}$and $\mathbf{Z}^{+}$is the set of all positive integers.

More precisely, if the solution $x(t)$ of (1.1) satisfies $x(t)=-x(t-2 N)$, let

$$
x_{1}(t)=x(t), \quad x_{2}(t)=x(t-1), \quad \ldots, \quad x_{2 N}(t)=x(t-(2 N-1)),
$$

then $X(t)=\left(x_{1}(t), x_{2}(t), \ldots, x_{2 N}(t)\right)^{T}$ satisfies

$$
\frac{d}{d t} X(t)=A_{2 N} G(t, X), \quad \text { where } A_{2 N}=\left(\begin{array}{cccc}
0 & -1 & \cdots & -1 \\
1 & 0 & \ddots & \vdots \\
\vdots & \ddots & \ddots & -1 \\
1 & \cdots & 1 & 0
\end{array}\right) \text {, }
$$

i.e., $A_{2 N}$ is a $2 N \times 2 N$ skew symmetric matrix, and

$$
G(t, X)=\left(f\left(t, x_{1}\right), f\left(t, x_{2}\right), \ldots, f\left(t, x_{2 N}\right)\right)^{T} .
$$

Also

$$
X(t)=\left(x_{1}(t), x_{2}(t), \ldots, x_{2 N}(t)\right)^{T}
$$

satisfies the following symmetric structure:

$$
\begin{aligned}
& x_{1}(t)=-x_{2 N}(t-1), \quad x_{2}(t)=x_{1}(t-1), \quad x_{3}(t)=x_{2}(t-1), \quad \ldots, \\
& x_{2 N}(t)=x_{2 N-1}(t-1) .
\end{aligned}
$$

In this paper, we have the following conditions on $f$.

(f1) There exist two $2 N$-periodic and continuous functions $\alpha, \beta$ such that

$$
\alpha(t)=\lim _{x \rightarrow 0} \frac{f(t, x)}{x}, \quad \beta(t)=\lim _{x \rightarrow \infty} \frac{f(t, x)}{x}
$$

uniformly for $t \in[0,2 N]$. 
$\left(\mathrm{f} 2^{ \pm}\right)$There exist $d_{1}, d_{2}>0$ such that

$$
\pm[2 F(t, x)-x f(t, x)] \geq d_{1}|x|-d_{2}, \quad(t, x) \in[0,2 N] \times \mathbf{R},
$$

where $F(t, x)=\int_{0}^{x} f(t, s) d s$.

Denote $m^{-}(t)=1$ if all $t<0 ; m^{-}(t)=0$ otherwise.

Theorem 1.1 Assume that $f$ satisfies (f1)-(f2-) with

$$
\beta(t)>\frac{\pi}{2 N} \tan \frac{\pi}{4 N}, \quad \text { for } t \in[0,2 N] .
$$

Then (1.1) possesses at least $2 m$ pairs $4 N$-periodic solutions with $x(t)=-x(t-2 N)$, where

$$
m=\sum_{j=1}^{\infty} m^{-}\left[\frac{\pi(2 j-1)}{2 N} \tan \frac{\pi(2 j-1)}{4 N}-\beta(t)\right],
$$

for

$$
\tan \frac{\pi(2 j-1)}{4 N}>0, \quad j \in \mathbf{Z}^{+} \text {and for all } t \in[0,2 N]
$$

Theorem 1.2 Assume that $f$ satisfies (f1)-(f2 $\left.{ }^{+}\right)$with

$$
\beta(t)<\frac{\pi(4 N-1)}{2 N} \tan \frac{\pi(4 N-1)}{4 N}, \text { for } t \in[0,2 N] .
$$

Then (1.1) possesses at least $2 m$ pairs $4 N$-periodic solutions with $x(t)=-x(t-2 N)$, where

$$
m=\sum_{j=1}^{\infty} m^{-}\left[\beta(t)-\frac{\pi(2 j-1)}{2 N} \tan \frac{\pi(2 j-1)}{4 N}\right],
$$

for

$$
\tan \frac{\pi(2 j-1)}{4 N}<0, \quad j \in \mathbf{Z}^{+} \text {and for all } t \in[0,2 N] .
$$

In this paper, the main purpose is to study the multiplicity of periodic solutions for the systems (1.1) via some recent critical point theorems for strongly indefinite functionals. In order to achieve this, some preliminaries are necessary. Let $X$ and $Y$ be Banach spaces with $X$ being separable and reflexive, and set $E=X \oplus Y$. Let $\mathcal{S} \subset X^{*}$ be a dense subset. For each $s \in \mathcal{S}$, there is a semi-norm on $E$ defined by

$$
p_{s}: E \rightarrow \mathbf{R}, \quad p_{s}(u)=|s(x)|+\|y\| \quad \text { for } u=x+y \in X \oplus Y .
$$

We denote by $\mathcal{T}_{\mathcal{S}}$ the topology on $E$ induced by a semi-norm family $\left\{p_{s}\right\}$, and let $\omega$ and $\omega^{*}$ denote the weak topology and weak* topology, respectively. Clearly, the $\mathcal{T}_{\mathcal{S}}$ topology contains the product topology on $E=X \oplus Y$ produced by the weak topology on $X$ and the strong topology on $Y$.

For a functional $\Phi \in C^{1}(E, \mathbf{R})$ we write $\Phi_{a}=\{u \in E: \Phi(u) \geq a\}$. Recall that $\Phi^{\prime}$ is weakly sequentially continuous if $u_{k} \rightarrow u$ in $E$, and one has $\lim _{k \rightarrow \infty} \Phi^{\prime}\left(u_{k}\right) v \rightarrow \Phi^{\prime}\left(u_{k}\right) v$ for each 
$v \in E$, i.e. $\Phi^{\prime}:(E, \omega) \rightarrow\left(E^{*}, \omega^{*}\right)$ is sequentially continuous. For $c \in \mathbf{R}$ we say that $\Phi$ satisfies the $(C)_{c}$ condition if any sequence $\left\{u_{k}\right\} \subset E$ such that $\Phi\left(u_{k}\right) \rightarrow c$ and $\left(1+\left\|u_{k}\right\|\right) \Phi^{\prime}\left(u_{k}\right) \rightarrow 0$ as $k \rightarrow \infty$ contains a convergent subsequence.

Suppose that

$\left(\Phi_{0}\right) \Phi \in C^{1}(E, \mathbf{R}), \Phi_{c}$ is $\mathcal{T}_{\mathcal{S}}$-closed for every $c \in \mathbf{R}$, and $\Phi^{\prime}:\left(\Phi_{c}, \mathcal{T}_{\mathcal{S}}\right) \rightarrow\left(E^{*}, \omega^{*}\right)$ is continuous.

$\left(\Phi_{1}\right)$ There exists a $\rho>0$ such that $\kappa:=\inf \Phi\left(B_{\rho} \cap Y\right)>0=\Phi(0)$, where $B_{\rho}=\{u \in E$ : $\|u\|=\rho\}$.

$\left(\Phi_{2}\right)$ There exist a finite dimensional subspace $Y_{0} \subset Y$ and $R>\rho$ such that $\bar{c}:=\sup \Phi\left(E_{0}\right)<$ $\infty$ and $\sup \Phi\left(E_{0} \backslash S_{0}\right)<\inf \Phi\left(B_{\rho} \cap Y\right)$, where $E_{0}:=X \oplus Y_{0}$, and $S_{0}=\left\{u \in E_{0}:\|u\| \leq R\right\}$.

The following critical point theorem will be used later (see $[1,5,15])$.

Theorem A Assume that $\Phi$ is even and $\left(\Phi_{0}\right)-\left(\Phi_{2}\right)$ are satisfied. Then $\Phi$ has at least $m=$ $\operatorname{dim} Y_{0}$ pairs of critical points with critical values less than or equal to $\bar{c}$ provided $\Phi$ satisfies the $(C)_{c}$ condition for all $c \in[\kappa, \bar{c}]$.

\section{Preliminaries}

It is easy to see that $A_{2 N}$ is a nonsingular skew symmetric Hamiltonian matrix, and (1.3) becomes a classical Hamiltonian system,

$$
z^{\prime}(t)=A_{2 N} \nabla H(t, z)
$$

where $\nabla H(t, z)$ denotes the gradient of $H(t, z)$ with respect to the $z$ variable, and

$$
H(t, z)=F\left(t, z_{1}\right)+F\left(t, z_{2}\right)+\cdots+F\left(t, z_{2 N}\right)
$$

for any $z=\left(z_{1}, z_{2}, \ldots, z_{2 N}\right)^{T} \in \mathbf{R}^{2 N}$.

For $S^{1}=\mathbf{R} /(4 N \mathbf{Z})$, let $H^{1 / 2}=W^{1 / 2,2}\left(S^{1}, \mathbf{R}^{2 N}\right)$. Then $H^{1 / 2}$ is a Hilbert space with norm $\|\cdot\|$ and inner product $\langle$,$\rangle , and H^{1 / 2}$ consists of those $z(t)$ in $L^{2}\left(S^{1}, \mathbf{R}^{2 N}\right)$ whose Fourier series,

$$
z(t)=a_{0}+\sum_{j=1}^{\infty}\left[a_{j} \cos \left(\frac{\pi}{2 N} j t\right)+a_{j} \cos \left(\frac{\pi}{2 N} j t\right)\right],
$$

satisfies

$$
\|z\|=4 N\left|a_{0}\right|^{2}+2 N \sum_{j=1}^{\infty} j\left(\left|a_{j}\right|^{2}+\left|b_{j}\right|^{2}\right)<\infty
$$

where $a_{j}, b_{j} \in \mathbf{R}^{2 N}$. By Proposition 6.6 [4], we know that $H^{1 / 2}$ is compactly embedded in $L^{s}\left(S^{1}, \mathbf{R}^{2 N}\right)$, where $s \in[1, \infty)$.

Since $A_{2 N}$ is a nonsingular skew, so is $A_{2 N}^{-1}$. We define an operator $L$ by extending the bilinear form

$$
\langle L z, y\rangle=\int_{0}^{4 N}\left(-A_{2 N}^{-1} \dot{z}(t), y(t)\right) d t .
$$

By direct computation, $L$ is a bounded self-adjoint linear operator on $H^{1 / 2}$. 
By (f1), one can show that $H(t, z) \in C^{1}\left(\mathbf{R} \times \mathbf{R}^{2 N}, \mathbf{R}\right)$ and there exist two continuous functions $a(t)>0, b(t) \geq 0$ such that

$$
H(t, z) \leq a(t)|z|^{2}+b(t), \quad \forall z \in \mathbf{R}^{2 N}
$$

By using similar arguments as in [4], Chapter 6, let

$$
\varphi(z)=\frac{1}{2}\langle L z, z\rangle+\psi(z)
$$

where

$$
\psi(z)=\int_{0}^{4 N} H(t, z(t)) d t
$$

Thus the critical points of $\varphi(z)$ in $H^{1 / 2}$ are classical solutions of (2.1).

Let $T_{2 N}$ be the $2 N \times 2 N$ matrix given

$$
T_{2 N}=\left(\begin{array}{ccccc}
0 & 0 & \cdots & 0 & -1 \\
1 & 0 & \ddots & 0 & 0 \\
0 & 1 & \ddots & \vdots & \vdots \\
\vdots & \ddots & \ddots & 0 & 0 \\
0 & \ldots & 0 & 1 & 0
\end{array}\right)
$$

For $z(t) \in H^{1 / 2}$, define by

$$
\delta z(t)=T_{2 N} z(t-1)
$$

Then $\delta^{2 N} z(t)=-z(t-2 N), \delta^{4 N} z(t)=z(t)$, and $G=\left\{\delta, \delta^{2}, \ldots, \delta^{4 N}\right\}$ is a compact group action over $H^{1 / 2}$. Moreover, if $\delta z(t)=z(t)$ holds, $z(t)$ has the symmetric structure (1.4).

Lemma 2.1 [11] Denote $E=\left\{z(t) \in H^{1 / 2}: \delta z(t)=z(t)\right\}$. Then

$$
\begin{aligned}
E= & \left\{z(t) \in H^{1 / 2}\left(S^{1}, \mathbf{R}^{2 N}\right): z(t)=\sum_{j=1}^{\infty}\left[a_{j} \cos \left(\frac{\pi}{2 N}(2 j-1) t\right)+b_{j} \sin \left(\frac{\pi}{2 N}(2 j-1) t\right)\right]:\right. \\
& \left.\left(\begin{array}{c}
a_{j} \\
b_{j}
\end{array}\right) \in \operatorname{span}\left\{\left(\begin{array}{c}
u_{j} \\
w_{j}
\end{array}\right),\left(\begin{array}{c}
-w_{j} \\
u_{j}
\end{array}\right)\right\}\right\},
\end{aligned}
$$

where $\beta_{j}=\frac{\pi}{2 N}(2 j-1)$ and

$$
\begin{aligned}
& u_{j}=\left(1, \cos \beta_{j}, \cos \left(2 \beta_{j}\right), \ldots, \cos (2 N-1) \beta_{j}\right)^{T}, \\
& w_{j}=\left(0, \sin \beta_{j}, \sin \left(2 \beta_{j}\right), \ldots, \sin (2 N-1) \beta_{j}\right)^{T} .
\end{aligned}
$$

Then we have the following lemma by using similar arguments [11].

Lemma 2.2 If $z(t)$ is a critical point of $\varphi$ in $E$, then $z(t)$ is a critical point of $\varphi$ in $H^{1 / 2}$. 
Proof By (2.2) and direct computation, we have

$$
A_{2 N} T_{2 N}=T_{2 N} A_{2 N}, \quad H\left(t, T_{2 N} z\right)=H(t, z), \quad \nabla H\left(t, T_{2 N} z\right)=T_{2 N} \nabla H(t, z) .
$$

Combing these with (2.6) and the fact that any $z(t) \in E$ is $4 N$-periodic, one can easily verify that

$$
\varphi(\delta z)=\varphi(z), \quad \varphi^{\prime}(\delta z)=\delta \varphi^{\prime}(z)
$$

i.e., $\varphi$ is G-invariant, and $\varphi^{\prime}$ is G-equivariant. The conclusion follows directly.

Remark 2.1 By Lemma 2.2, the function $\varphi$ is invariant and $\varphi$ is equivariant about the compact group $G$. This allows to find critical points of $\varphi$ on a subspace of $E$ which is invariant under the group $G$. Then we can apply Theorem A to obtain periodic solutions in this subspace, which surely have the required symmetric structure and give solutions to (1.1).

Moreover, we also denote by $M^{+}(\cdot), M^{-}(\cdot)$ and $M^{0}(\cdot)$ the positive definite, negative definite, and null subspaces of the self-adjoint linear operator defining it, respectively.

Then $E$ has an orthogonal decomposition

$$
E=M^{+}(L) \oplus M^{-}(L) \oplus M^{0}(L) .
$$

By Lemma $2.3[11]$, for $z_{j}(t)=a_{j} \cos \left(\frac{\pi}{2 N}(2 j-1) t\right)+b_{j} \sin \left(\frac{\pi}{2 N}(2 j-1) t\right)(j=1,2, \ldots), a_{j}$ and $b_{j}$ are defined in Lemma 2.1, we have

$$
\left\langle L z_{j}, z_{j}\right\rangle=-\frac{\pi(2 j-1)}{2 N} \tan \frac{\pi(2 j-1)}{4 N}\left[\left(a_{j}, a_{j}\right)+\left(b_{j}, b_{j}\right)\right] .
$$

Let

$$
\sigma=\min \left\{\left|\frac{\pi(2 j-1)}{2 N} \tan \frac{\pi(2 j-1)}{4 N}\right|: j \in Z^{+}\right\}>0 .
$$

So we have

$$
\begin{aligned}
& \left\langle L z_{j}, z_{j}\right\rangle \geq \sigma\left\|z_{j}\right\|^{2}, \quad \text { for } \gamma_{j}>0, \\
& \left\langle L z_{j}, z_{j}\right\rangle \leq-\sigma\left\|z_{j}\right\|^{2}, \quad \text { for } \gamma_{j}<0,
\end{aligned}
$$

where $\gamma_{j}=-\tan \frac{\pi(2 j-1)}{4 N}$. Therefore, we get

$$
\begin{aligned}
& \langle L z, z\rangle \geq \sigma\|z\|^{2}, \quad \text { for } z \in M^{+}(L), \\
& \langle L z, z\rangle \leq-\sigma\|z\|^{2}, \quad \text { for } z \in M^{-}(L) .
\end{aligned}
$$

\section{Proofs of theorems}

In this section, $c_{i}$ stand for different positive constants for $i \in Z^{+}$. 
By direct computation, (f1) implies that $H(t, z)$ is even and satisfies

$$
\begin{aligned}
& \nabla H(t, z)=\alpha(t) z+o(|z|) \quad \text { as }|z| \rightarrow 0, \\
& \nabla H(t, z)=\beta(t) z+o(|z|) \quad \text { as }|z| \rightarrow \infty,
\end{aligned}
$$

uniformly for $t \in[0,2 N]$.

$\left(\mathrm{f} 2^{ \pm}\right)$implies that

$$
2 H(t, z)-(\nabla H(t, z), z) \geq d_{1}|z|-2 N d_{2}, \quad \forall(t, z) \in[0,2 N] \times \mathbf{R}^{2 N}
$$

and

$$
-[2 H(t, z)-(\nabla H(t, z), z)] \geq d_{1}|z|-2 N d_{2}, \quad \forall(t, z) \in[0,2 N] \times \mathbf{R}^{2 N} .
$$

Remark 3.1 Here we prove that (3.3) and (3.4) hold. By (2.2) and (f2 $\left.{ }^{ \pm}\right)$, we have

$$
\begin{aligned}
\pm[2 H(t, z)-(\nabla H(t, z), z)] & = \pm \sum_{i=1}^{2 N}\left[2 F\left(t, z_{i}\right)-z_{i} f\left(t, z_{i}\right)\right] \\
& \geq d_{1} \sum_{i=1}^{2 N}\left|z_{i}\right|-2 N d_{2} \geq d_{1}|z|-2 N d_{2} .
\end{aligned}
$$

Lemma 3.1 Suppose that $f$ satisfies $(\mathrm{f} 1)-\left(\mathrm{f} 2^{ \pm}\right)$. Then the function $\varphi$ satisfies the $(\mathrm{C})_{c}$ condition for any $c \in \mathbf{R}$.

Proof First, we need some notations. For any $z, y \in E$, define an operator $B$ by extending the bilinear form

$$
\langle B z, y\rangle=\int_{0}^{4 N}(\beta(t) z(t), y(t)) d t .
$$

By direct computation, $B$ is a bounded self-adjoint linear operator on $E$. Thus $L+B$ is also a self-adjoint linear operator on $E$.

Let

$$
E=M^{+}(L+B) \oplus M^{-}(L+B) \oplus M^{0}(L+B) .
$$

Also there exists $\sigma_{1}>0$ such that

$$
\begin{aligned}
& \langle(L+B) z, z\rangle \geq \sigma_{1}\|z\|^{2}, \quad \text { for } z \in M^{+}(L+B), \\
& \langle(L+B) z, z\rangle \leq-\sigma_{1}\|z\|^{2}, \quad \text { for } z \in M^{-}(L+B) .
\end{aligned}
$$

So we have

$$
\Phi(z)=-\varphi(z)=-\frac{1}{2}\langle(L+B) z, z\rangle-\int_{0}^{4 N}\left[H(t, z)-\frac{1}{2}(\beta(t) z, z)\right] d t
$$

and

$$
\left\langle\Phi^{\prime}(z), y\right\rangle=-\langle(L+B) z, y\rangle-\int_{0}^{4 N}(\nabla H(t, z)-\beta(t) z, y) d t .
$$


Let $\left\{z_{k}\right\} \subset E$ be any sequence such that

$$
\Phi\left(z_{k}\right) \rightarrow c, \quad\left(1+\left\|z_{k}\right\|\right) \Phi^{\prime}\left(z_{k}\right) \rightarrow 0 \quad \text { as } k \rightarrow \infty .
$$

We first prove that $\left\{z_{k}\right\}$ is bounded. Since $z_{k} \in E$, we have

$$
z_{k}=z_{k}^{+}+z_{k}^{-}+z^{0} \in M^{+}(L+B) \oplus M^{-}(L+B) \oplus M^{0}(L+B) .
$$

Let

$$
h(z)=\int_{0}^{4 N} \tilde{H}(t, z) d t, \quad \tilde{H}(t, z)=H(t, z)-\frac{1}{2}(\beta(t) z, z), \quad z \in E .
$$

By (3.2), we have

$$
\frac{|\nabla \tilde{H}(t, z)|}{|z|} \rightarrow 0 \quad \text { as }|z| \rightarrow \infty,
$$

uniformly for $t \in[0,2 N]$. This means that, for any $\varepsilon>0$, there exists $M>0$ such that

$$
|\nabla \tilde{H}(t, z)| \leq \varepsilon|z|+M, \quad \text { for all }(t, z) \in[0,2 N] \times \mathbf{R}^{2 N} .
$$

Therefore, for any $y \in E$,

$$
\begin{aligned}
\left|\left\langle h^{\prime}\left(z_{k}\right), y\right\rangle\right| & =\left|\int_{0}^{4 N}\left(\nabla \tilde{H}\left(t, z_{k}(t)\right), y(t)\right) d t\right| \\
& \leq \int_{0}^{4 N}\left|\nabla \tilde{H}\left(t, z_{k}(t)\right)\right||y(t)| d t \\
& \leq\left(\int_{0}^{4 N}\left|\nabla \tilde{H}\left(t, z_{k}(t)\right)\right|^{2} d t\right)^{1 / 2}\|y\|_{L^{2}} \\
& \leq\left(2 \varepsilon^{2}\left\|z_{k}\right\|_{L^{2}}^{2}+8 N M^{2}\right)^{1 / 2}\|y\|_{L^{2}} \\
& \leq\left(2 \varepsilon^{2}\left\|z_{k}\right\|^{2}+8 N M^{2}\right)^{1 / 2}\|y\| .
\end{aligned}
$$

This implies

$$
\lim _{k \rightarrow \infty} \frac{\left\|h^{\prime}\left(z_{k}\right)\right\|}{\left\|z_{k}\right\|} \leq \sqrt{2} \varepsilon, \quad \text { for any } \varepsilon>0,
$$

i.e.

$$
\frac{\left\|h^{\prime}\left(z_{k}\right)\right\|}{\left\|z_{k}\right\|} \rightarrow 0 \quad \text { as } k \rightarrow \infty
$$

Then

$$
\begin{aligned}
\left\langle-\Phi^{\prime}\left(z_{k}\right), z_{k}^{+}\right\rangle & =\left\langle(L+B) z_{k}^{+}, z_{k}^{+}\right\rangle+\left\langle h^{\prime}\left(z_{k}\right), z_{k}^{+}\right\rangle \\
& \geq \sigma_{1}\left\|z_{k}^{+}\right\|^{2}-\left\|h^{\prime}\left(z_{k}\right)\right\|\left\|z_{k}^{+}\right\| .
\end{aligned}
$$


By (3.7) and (3.9), we have

$$
\frac{\left\|z_{k}^{+}\right\|}{\left\|z_{k}\right\|} \rightarrow 0 \quad \text { as } k \rightarrow \infty
$$

Similarly,

$$
\frac{\left\|z_{k}^{-}\right\|}{\left\|z_{k}\right\|} \rightarrow 0 \quad \text { as } k \rightarrow \infty
$$

Case (i): (3.3) holds. We have

$$
\begin{aligned}
& \left\langle\Phi^{\prime}\left(z_{k}\right), z_{k}\right\rangle-2 \Phi\left(z_{k}\right) \\
& \quad=\int_{0}^{4 N}\left[2 H\left(t, z_{k}\right)-\left(\nabla H\left(t, z_{k}\right), z_{k}\right)\right] d t \\
& \quad \geq d_{1} \int_{0}^{4 N}\left|z_{k}\right| d t-8 N^{2} d_{2} \\
& \quad \geq d_{1} \int_{0}^{4 N}\left|z_{k}^{0}\right| d t-d_{1} \int_{0}^{4 N}\left(\left|z_{k}^{+}\right|+\left|z_{k}^{-}\right|\right) d t-8 N^{2} d_{2} \\
& \quad \geq d_{1}\left\|z_{k}^{0}\right\|-d_{1}\left(\left\|z_{k}^{+}\right\|+\left\|z_{k}^{-}\right\|\right)-8 N^{2} d_{2} .
\end{aligned}
$$

Here we used the fact that $M^{0}(L+B)$ is finite dimensional. By (3.8), (3.10), and (3.11), we have

$$
\frac{\left\|z_{k}^{0}\right\|}{\left\|z_{k}\right\|} \rightarrow 0 \quad \text { as } k \rightarrow \infty
$$

But this implies the following contradiction:

$$
1=\frac{\left\|z_{k}\right\|}{\left\|z_{k}\right\|} \leq \frac{\left\|z_{k}^{0}\right\|+\left\|z_{k}^{-}\right\|+\left\|z_{k}^{+}\right\|}{\left\|z_{k}\right\|} \rightarrow 0 \quad \text { as } k \rightarrow \infty .
$$

Therefore $\left\{\left\|z_{k}\right\|\right\}$ must be bounded.

Case (ii): (3.4) holds. Similar to Case (i), we have

$$
\begin{aligned}
& \left\langle 2 \Phi\left(z_{k}\right)-\Phi^{\prime}\left(z_{k}\right), z_{k}\right\rangle \\
& \quad=\int_{0}^{4 N}\left[\left(\nabla H\left(t, z_{k}\right), z_{k}\right)-2 H\left(t, z_{k}\right)\right] d t \\
& \quad \geq d_{1} \int_{0}^{4 N}\left|z_{k}\right| d t-8 N^{2} d_{2} \\
& \quad \geq d_{1} \int_{0}^{4 N}\left|z_{k}^{0}\right| d t-d_{1} \int_{0}^{4 N}\left(\left|z_{k}^{+}\right|+\left|z_{k}^{-}\right|\right) d t-8 N^{2} d_{2} \\
& \quad \geq d_{1}\left\|z_{k}^{0}\right\|-d_{1}\left(\left\|z_{k}^{+}\right\|+\left\|z_{k}^{-}\right\|\right)-8 N^{2} d_{2} .
\end{aligned}
$$

This implies (3.12) and (3.13). Thus $\left\{\left\|z_{k}\right\|\right\}$ must be bounded. 
So we see that $\left\{z_{k}\right\}$ is bounded, and going if necessary to a subsequence, we can assume that $z_{k} \rightarrow z$ in $E$ and $z_{k} \rightarrow z$ in $L\left(S^{1}, \mathbf{R}^{2 N}\right)$. Write $z_{k}=z_{k}^{+}+z_{k}^{-}+z_{k}^{-}$and $z=z^{+}+z^{-}+z^{0}$, then $z_{k}^{ \pm} \rightarrow z^{ \pm}$in $E, z_{k}^{0} \rightarrow z^{0}$ in $E$, and $z_{k}^{ \pm} \rightarrow z^{ \pm}$in $L\left(S^{1}, \mathbf{R}^{2 N}\right)$.

In view of (3.8) and $z_{k}^{-} \rightarrow z^{-}$in $L\left(S^{1}, \mathbf{R}^{2 N}\right)$, it is easy to verify

$$
\int_{0}^{4 N}\left(\nabla H\left(t, z_{k}\right)-\beta(t) z_{k}, z_{k}^{-}-z^{-}\right) d t \rightarrow 0
$$

and

$$
\int_{0}^{4 N}\left(\nabla H(t, z)-\beta(t) z, z_{k}^{-}-z^{-}\right) d t \rightarrow 0
$$

But then $\left\langle\Phi^{\prime}\left(z_{k}\right)-\Phi^{\prime}(z), z_{k}^{-}-z^{-}\right\rangle \rightarrow 0$ as $k \rightarrow \infty$, and

$$
\begin{aligned}
& \left\langle\Phi^{\prime}\left(z_{k}\right)-\Phi^{\prime}(z), z_{k}^{-}-z^{-}\right\rangle \\
& =-\left\langle(L+B)\left(z_{k}^{-}-z^{-}\right), z_{k}^{-}-z^{-}\right\rangle \\
& \quad-\int_{0}^{4 N}\left(\nabla H\left(t, z_{k}\right)-\beta(t) z_{k}, z_{k}^{-}-z^{-}\right) d t+\int_{0}^{4 N}\left(\nabla H(t, z)-\beta(t) z, z_{k}^{-}-z^{-}\right) d t \\
& \geq \sigma_{1}\left\|z_{k}^{-}-z^{-}\right\|^{2}-\int_{0}^{4 N}\left(\nabla H\left(t, z_{k}\right)-\beta(t) z_{k}, z_{k}^{-}-z^{-}\right) d t \\
& \quad+\int_{0}^{4 N}\left(\nabla H(t, z)-\beta(t) z, z_{k}^{-}-z^{-}\right) d t .
\end{aligned}
$$

This yields $z_{k}^{-} \rightarrow z^{-}$in $E$. Similarly, $z_{k}^{+} \rightarrow z^{+}$in $E$ and hence $z_{k} \rightarrow z$ in $E$, that is, $\Phi$ satisfies the $(C)_{c}$ condition. Thus $\varphi$ satisfies the $(C)_{c}$ condition. The proof of Lemma 3.1 is complete.

Proof of Theorem 1.1 Let $X=M^{+}(L) \oplus M^{0}(L), Y=M^{-}(L), E=X \oplus Y$, and

$$
\Phi(z)=-\varphi(z), \quad \Psi(z)=\psi(z)
$$

where $\varphi(z)$ and $\psi(z)$ are defined in Section 2.

In order to obtain this theorem, we will apply Theorem A to the functional $\Phi(z)$. Let $\mathcal{S}=X^{*}, \mathcal{T}_{\mathcal{S}}=E=X \oplus Y$. The proof of this theorem is divided into the following three steps.

Step 1. $\Phi$ satisfies $\left(\Phi_{0}\right)$.

We first check that $\Phi_{c}$ is $\mathcal{T}_{\mathcal{S}}$-closed for any $c \in \mathbf{R}$. Let $\left\{z_{k}\right\}$ be any sequence $\mathcal{T}_{\mathcal{S}}$-converging to some $z \in E$. Write $z_{k}=z_{k}^{+}+z_{k}^{0}+z_{k}^{-}$and $z=z^{+}+z^{0}+z^{-}$, then $z_{k}^{-} \rightarrow u^{-}$in $E$ and hence $\left\{z^{-}\right\}$is bounded in the norm topology.

Note that $\beta(t)>\frac{\pi}{2 N} \tan \frac{\pi}{4 N}$ for $t \in[0,2 N]$, then for any $z \in E$ and small $\varepsilon>0$, by (3.8), we have

$$
\begin{aligned}
\Psi(z) & =\int_{0}^{4 N} H(t, z) d t=\int_{0}^{4 N} \int_{0}^{1}(\nabla H(t, s z), z) d s d t \\
& \geq \int_{0}^{4 N} \int_{0}^{1}(\beta(t) s z, z) d s d t
\end{aligned}
$$




$$
\begin{aligned}
& -\int_{0}^{4 N} \int_{0}^{1}|\nabla H(t, s z)-(\beta(t) s z, z)||z| d s d t \\
\geq & \frac{1}{2} \int_{0}^{4 N}(\beta(t) z, z) d t-\int_{0}^{4 N} \int_{0}^{1}(\varepsilon|s z|+M)|z| d s d t \\
\geq & \frac{1}{2}\left(\frac{\pi}{2 N} \tan \frac{\pi}{4 N}-\varepsilon\right)\|z\|_{L^{2}}^{2}-M\|z\|_{L^{2}} \geq c_{1}\|z\|_{L^{2}}^{2}-c_{2}\|z\|_{L^{2}},
\end{aligned}
$$

which implies that $\Psi$ is bounded from below on $E$. Consequently, combining $z_{k} \in \Phi_{c}$ and $\frac{1}{2}\left\langle L z_{k}^{+}, z_{k}^{+}\right\rangle=-\frac{1}{2}\left\langle L z_{k}^{-}, z_{k}^{-}\right\rangle-\Phi\left(z_{k}\right)-\Psi\left(z_{k}\right)$ shows that $\left\{z_{k}^{+}\right\}$is bounded in $E$ by (2.7) and hence

$$
\begin{aligned}
\Psi\left(z_{k}\right) & =-\frac{1}{2}\left\langle L z_{k}^{+}, z_{k}^{+}\right\rangle-\frac{1}{2}\left\langle L z_{k}^{-}, z_{k}^{-}\right\rangle-\Phi\left(z_{k}\right) \\
& \leq-\frac{1}{2}\left\langle L z_{k}^{+}, z_{k}^{+}\right\rangle-\frac{1}{2}\left\langle L z_{k}^{-}, u_{k}^{-}\right\rangle-c \leq c_{3} .
\end{aligned}
$$

Moreover, since $\left\|z_{k}\right\|_{L^{2}}^{2}=\left\|z_{k}^{+}\right\|_{L^{2}}^{2}+\left\|z_{k}^{0}\right\|_{L^{2}}^{2}+\left\|z_{k}^{-}\right\|_{L^{2}}^{2}$, we have

$$
\Psi\left(z_{k}\right) \geq c_{1}\left\|z_{k}\right\|_{L^{2}}^{2}-c_{2}\left\|z_{k}\right\|_{L^{2}} \geq\left\|z_{k}\right\|_{L^{2}}-c_{4} \geq\left\|z_{k}^{0}\right\|_{L^{2}}-c_{4} .
$$

It follows from (3.14) and (3.15) that $\left\{z_{k}^{0}\right\}$ is also bounded in $E$ since all norms are equivalent in a finite dimensional space. Then $\left\|z_{k}\right\|^{2}$ is bounded, and hence we can assume that $\left\{z_{k}\right\}$ converges weakly to $z=z^{+}+z^{0}+z^{-}$in $E$. Thus we have $\Psi\left(z_{k}\right) \rightarrow \Psi(z)$. Note that $\langle L y, y\rangle^{1 / 2}$ is an equivalent norm on $\mathrm{H}^{+}$. By the lower semi-continuity of the norm, we get

$$
\begin{aligned}
c & \leq \limsup _{k \rightarrow \infty} \Phi\left(z_{k}\right)=\limsup _{k \rightarrow \infty}\left(-\frac{1}{2}\left\langle L z_{k}^{+}, z_{k}^{+}\right\rangle-\frac{1}{2}\left\langle L z_{k}^{-}, z_{k}^{-}\right\rangle-\Psi\left(z_{k}\right)\right) \\
& =-\liminf _{k \rightarrow \infty} \frac{1}{2}\left\langle L z_{k}^{+}, z_{k}^{+}\right\rangle-\frac{1}{2}\left\langle L z^{-}, z^{-}\right\rangle-\Psi(z) \leq \Phi(z),
\end{aligned}
$$

that is, $z \in \Phi_{c}$ and hence $\Phi_{c}$ is $\mathcal{T}_{\mathcal{S}}$-closed.

Next, we prove that $\Phi^{\prime}:\left(\Phi_{c}, \mathcal{T}_{\mathcal{S}}\right) \rightarrow\left(E^{*}, \omega^{*}\right)$ is continuous. To achieve this, it is sufficient to demonstrate that $\Psi^{\prime}$ has the same property. Suppose $z_{k} \rightarrow u$ in $E$, then $\left\{z_{k}\right\}$ converges uniformly to $z$ on $[0,4 N]$. Hence, for every given $y \in E$, we see that $\left(\nabla H\left(t, z_{k}(t)\right), y(t)\right)$ converges to $(\nabla H(t, z(t)), y(t))$ in measure on $[0,4 N]$. Moreover, by (3.8), one has

$$
\left|\left(\nabla H\left(t, z_{k}(t)\right), y(t)\right)\right| \leq\left(\varepsilon\left\|z_{k}\right\|_{\infty}+\bar{\beta}\left\|z_{k}\right\|_{\infty}+M\right)\|y\|_{\infty} \leq c_{5}
$$

for all $k$ and $t \in[0,4 N]$, where $\bar{\beta}=\max _{t \in[0,2 N]}\{\beta(t)\}$ and $\|\cdot\|_{\infty}$ denotes the natural norm of $C\left(S^{1}, \mathbf{R}^{2 N}\right)$. Thus, the Vitali theorem is applicable and

$$
\left\langle\Psi^{\prime}\left(z_{k}\right), y\right\rangle=\int_{0}^{4 N}\left(\nabla H\left(t, z_{k}\right), y\right) d t \rightarrow \int_{0}^{4 N}(\nabla H(t, z), y) d t=\left\langle\Psi^{\prime}(z), y\right\rangle
$$

for any $y \in E$. So $\Phi$ satisfies $\left(\Phi_{0}\right)$.

Step 2. $\Phi$ satisfies $\left(\Phi_{1}\right)$.

By (3.1) and (3.2), for any $\varepsilon>0$, there exist $C_{\varepsilon}>0$ and $p>2$ such that

$$
H(t, z) \leq \varepsilon|z|^{2}+C_{\varepsilon}|z|^{p}, \quad \text { for all }(t, z) \in[0,4 N] \times \mathbf{R}^{2 N} .
$$


Hence, for $z \in Y$ and small $\varepsilon$, we have

$$
\begin{aligned}
\Phi(z) & =-\frac{1}{2}\langle L z, z\rangle-\int_{0}^{4 N} H(t, z) d t \\
& \geq-\frac{1}{2}\langle L z, z\rangle-\int_{0}^{4 N}\left(\varepsilon|z|^{2}+C_{\varepsilon}|z|^{p}\right) d t \\
& \geq \frac{\sigma}{2}\|z\|^{2}-\varepsilon\|z\|_{L^{2}}^{2}-C_{\varepsilon}\|z\|_{L^{p}}^{p} \\
& \geq \frac{\sigma}{2}\|z\|^{2}-\varepsilon \xi_{1}\|z\|^{2}-C_{\varepsilon} \xi_{2}\|z\|^{p} \\
& \geq \frac{\sigma}{4}\|z\|^{2}-c_{5}\|z\|^{p},
\end{aligned}
$$

where $\xi_{1}, \xi_{2}>0$ satisfy $\|z\|_{L^{2}} \leq \xi_{1}\|z\|$, $\|z\|_{L^{p}} \leq \xi_{2}\|z\|$; see [4], Proposition 6.6.

Since $p>2$, there exists a small $\rho>0$ such that $\frac{1}{8} \rho^{2} \geq c_{5} \rho^{p}$. Therefore,

$$
\kappa:=\inf \Phi\left(B_{\rho} \cap Y\right) \geq \frac{1}{8} \rho^{2}>0,
$$

and hence $\left(\Phi_{1}\right)$ holds.

Step 3. $\Phi$ satisfies $\left(\Phi_{2}\right)$.

Let

$$
\begin{aligned}
Y_{0}= & \operatorname{span}\left\{a_{j} \cos \left(\frac{\pi}{2 N}(2 j-1) t\right), b_{j} \sin \left(\frac{\pi}{2 N}(2 j-1) t\right):\right. \\
& \left.\beta(t)>\frac{\pi(2 j-1)}{2 N} \tan \frac{\pi(2 j-1)}{4 N}>0, \text { for all } t \in[0,2 N]\right\},
\end{aligned}
$$

where $a_{j}$ and $b_{j}$ are defined in Lemma 2.1.

Obviously, $Y_{0} \subset Y$ and $\operatorname{dim} Y_{0}=2 m$. In order to obtain the desired conclusion, it is sufficient to prove that $\Phi(z) \rightarrow-\infty$ as $\|z\| \rightarrow \infty$ on $E_{0}:=X \oplus Y_{0}$.

Let

$$
m_{1}=\max \left\{j \in \mathbf{Z}^{+}: \beta(t)>\frac{\pi(2 j-1)}{2 N} \tan \frac{\pi(2 j-1)}{4 N}>0 \text {, for all } t \in[0,2 N]\right\}
$$

and

$$
\Gamma=-\frac{\pi\left(2 m_{1}-1\right)}{2 N} \tan \frac{\pi\left(2 m_{1}-1\right)}{4 N} .
$$

By the definition of $\Gamma$, there exists a constant $0<\delta<\sigma ; \sigma$ is defined in (2.8), such that

$$
\beta(t) \geq-\Gamma+\delta
$$

for $t \in[0,2 N]$. Let

$$
J=\left\{j \in \mathbf{Z}^{+}: \beta(t)>\frac{\pi(2 j-1)}{2 N} \tan \frac{\pi(2 j-1)}{4 N}>0 \text {, for all } t \in[0,2 N]\right\} .
$$


For any $y \in Y_{0}$, by (2.7),

$$
\begin{aligned}
\langle L y, y\rangle & =\sum_{j \in J}\left(-\frac{\pi(2 j-1)}{2 N} \tan \frac{\pi(2 j-1)}{4 N}\left[\left(a_{j}, a_{j}\right)+\left(b_{j}, b_{j}\right)\right]\right) \\
& \geq \Gamma\|y\|_{L^{2}}^{2} .
\end{aligned}
$$

Let $\widetilde{H}(t, z)=H(t, z)-\frac{1}{2}(\beta(t) z, z)$. We claim that, for $0 \neq z \in E$,

$$
\|z\|^{-2} \int_{0}^{4 N} \tilde{H}(t, z) d t \rightarrow 0 \quad \text { as }\|z\| \rightarrow \infty .
$$

Indeed, for $0 \neq z \in E$, by (3.8), one has

$$
\begin{aligned}
& \|z\|^{-2}\left|\int_{0}^{4 N} \tilde{H}(t, z) d t\right| \\
& =\|z\|^{-2}\left|\int_{0}^{4 N} \int_{0}^{1}(\nabla H(t, s z)-\beta(t) s z, z) d s d t\right| \\
& \leq\|z\|^{-2} \int_{0}^{4 N}(\varepsilon|z|+M)|z| d t \\
& \leq\|z\|^{-2}\left(\varepsilon\|z\|_{L^{2}}^{2}+M\|z\|_{L^{2}}\right) \\
& \quad \leq \varepsilon+\frac{M}{\|z\|}
\end{aligned}
$$

which implies that (3.18) is true by the arbitrariness of $\varepsilon$. Then, for $z=z^{+}+z^{0}+z^{-} \in E_{0}$, by (3.16)-(3.17) and (2.8), one has

$$
\begin{aligned}
\Phi(z)= & -\frac{1}{2}\left\langle L z^{-}, z^{-}\right\rangle-\frac{1}{2}\left\langle L z^{+}, z^{+}\right\rangle-\int_{0}^{4 N} H(t, z) d t \\
\leq & -\frac{\Gamma}{2}\left\|z^{-}\right\|_{L^{2}}^{2}-\frac{\delta}{2}\left\|z^{+}\right\|^{2}-\frac{1}{2} \int_{0}^{4 N}(\beta(t) z, z) d t-\int_{0}^{4 N} \tilde{H}(t, z) d t \\
\leq & -\frac{\Gamma}{2}\left\|z^{-}\right\|_{L^{2}}^{2}-\frac{\delta}{2}\left\|z^{+}\right\|^{2}-\frac{1}{2}(-\Gamma+\delta)\|z\|_{L^{2}}^{2}-\int_{0}^{4 N} \tilde{H}(t, z) d t \\
\leq & -\frac{\Gamma}{2}\left\|z^{-}\right\|_{L^{2}}^{2}-\frac{\delta}{2}\left\|z^{+}\right\|^{2}-\frac{1}{2}(-\Gamma+\delta)\left(\left\|z^{-}\right\|_{L^{2}}^{2}+\left\|z^{0}\right\|_{L^{2}}^{2}\right) \\
& -\int_{0}^{4 N} \widetilde{H}(t, z) d t \\
\leq & -\frac{\delta}{2}\left(\left\|z^{-}\right\|_{L^{2}}^{2}+\left\|z^{+}\right\|^{2}+\left\|z^{0}\right\|_{L^{2}}^{2}\right)-\int_{0}^{4 N} \tilde{H}(t, z) d t .
\end{aligned}
$$

Since $M^{0}$ and $Y_{0}$ are finitely dimensional, (3.18) and the above estimate imply that $\Phi(z) \rightarrow$ $-\infty$ as $\|z\| \rightarrow \infty, z \in E_{0}:=X \oplus Y_{0}$. Hence $\left(\Phi_{2}\right)$ holds.

The proof of Theorem 1.1 is complete.

Proof of Theorem 1.2 Let $X=M^{-}(L) \oplus M^{0}(L), Y=M^{+}(L), E=X \oplus Y$, and

$$
\Phi(z)=\varphi(z), \quad \Psi(z)=-\psi(z)
$$


and

$$
\begin{array}{r}
Y_{0}=\operatorname{span}\left\{a_{j} \cos \left(\frac{\pi}{2 N}(2 j-1) t\right), b_{j} \sin \left(\frac{\pi}{2 N}(2 j-1) t\right):\right. \\
\left.\beta(t)<\frac{\pi(2 j-1)}{2 N} \tan \frac{\pi(2 j-1)}{4 N}<0, \text { for } t \in[0,2 N]\right\},
\end{array}
$$

where $a_{j}$ and $b_{j}$ are defined in Lemma 2.1.

Then the conclusion will be obtained by the same argument as in the proof of Theorem 1.1. The proof of Theorem 1.2 is complete.

Consider the nonautonomous delay equation

$$
x^{\prime}(t)=-f(t, x(t-1))
$$

Example 3.1 Consider the nonautonomous delay equation (3.19), where

$$
f(t, x)=a(t) x-b(t)\left[\frac{2 x}{\ln \left(e+x^{2}\right)}-\frac{2 x^{3}}{\left(e+x^{2}\right) \ln ^{2}\left(e+x^{2}\right)}\right]
$$

and $a(t)=\pi\left(2+\tan \left(\frac{\pi t}{4}-\frac{\pi}{4}\right)\right), b(t)=\frac{3 \pi}{2}$ for $t \in[0,2]$.

It is easy to see that $\alpha(t)=a(t)-2 b(t) \leq 0, \beta(t)=a(t) \geq \pi$ for $t \in[0,2]$, and $F(t, x)=$ $\int_{0}^{x} f(t, s) d s=\frac{a(t) x^{2}}{2}-\frac{b(t) x^{2}}{\ln \left(e+x^{2}\right)}$. By Theorem 1.1, we get $m=1$. Thus (3.19) possesses at least two pairs of 4-periodic solutions with $x(t)=-x(t-2)$.

Example 3.2 Consider the nonautonomous delay equation (3.19), where

$$
f(t, x)=a(t) x-b(t)\left[\frac{2 x}{\ln \left(e+x^{2}\right)}-\frac{2 x^{3}}{\left(e+x^{2}\right) \ln ^{2}\left(e+x^{2}\right)}\right]
$$

and $a(t)=4 \pi\left(-2+\tan \left(\frac{\pi t}{4}-\frac{\pi}{4}\right)\right), b(t)=-6 \pi$ for $t \in[0,2]$.

It is easy to see that $\alpha(t)=a(t)-2 b(t) \geq 0, \beta(t)=a(t) \leq-4 \pi$ for $t \in[0,2]$. By Theorem 1.2, we get $m=2$. Thus (3.19) possesses at least four pairs of 4-periodic solutions with $x(t)=-x(t-2)$.

\section{Competing interests}

The author declares that they have no competing interests.

\section{Acknowledgements}

The author is grateful for the referee's careful reviewing and helpful comments. The research was supported by the Natural Science Foundation of Shanxi Province of China (No. 2012011004-1) and by the National Natural Science Foundation of China (No. 61473180).

Received: 10 October 2014 Accepted: 6 April 2015 Published online: 30 April 2015

\section{References}

1. Bartsch, T, Ding, Y: Deformation theorems on non-metrizable vector spaces and applications to critical point theory. Math. Nachr. 279(12), 1267-1288 (2006)

2. Fannio, LO: Multiple periodic solutions of Hamiltonian systems with strong resonance at infinity. Discrete Contin. Dyn. Syst. 3, 251-264 (1997)

3. Mawhin, J, Willem, M: Critical Point Theory and Hamiltonian Systems. Springer, New York (1989)

4. Rabinowits, PH: Minimax Methods in Critical Point Theory with Applications to Differential Equations. CBMS, vol. 65. Am. Math. Soc., Providence (1986)

5. Su, JB: Nontrivial periodic solutions for the asymptotically linear Hamiltonian systems with resonance at infinity. J. Differ. Equ. 145, 252-273 (1998) 
6. Zhao, F, Zhao, L, Ding, Y: Existence and multiplicity of solutions for a non-periodic Schrödinger equation. Nonlinear Anal. TMA 69, 3671-3678 (2008)

7. $L i, J, H e, X$ : Multiple periodic solutions of differential delay equations created by asymptotically linear Hamiltonian systems. Nonlinear Anal. 31, $45-54$ (1998)

8. Guo, Z, Yu, J: Multiplicity results for periodic solutions to delay differential equations via critical point theory. J. Differ. Equ. 218, 15-35 (2005)

9. Cheng, R, Xu, J: Periodic solutions for a class of non-autonomous differential delay equations. Nonlinear Differ. Equ. Appl. 16, 793-809 (2009)

10. Fei, G: Nontrival periodic solutions of asymptotically linear Hamiltonian systems. Electron. J. Differ. Equ. 2001, 69 (2001)

11. Fei, G: Multiple periodic solutions of differential delay equations via Hamiltonian systems (I). Nonlinear Anal. 65, 25-39 (2006)

12. Fei, G: Multiple periodic solutions of differential delay equations via Hamiltonian systems (II). Nonlinear Anal. 65 40-58 (2006)

13. Guo, C, Guo, Z: Existence of multiple periodic solutions for a class of second-order delay differential equations. Nonlinear Anal., Real World Appl. 10, 3285-3297 (2009)

14. $\mathrm{Li}, \mathrm{J}, \mathrm{He}, \mathrm{X}, \mathrm{Liu}, \mathrm{Z}$ : Hamiltonian symmetric groups and multiple periodic solutions of differential delay equations. Nonlinear Anal. TMA 35, 457-474 (1999)

15. $\mathrm{Wu}, \mathrm{K}, \mathrm{Wu}, \mathrm{X}$ : Existence of periodic solutions for a class of first order delay differential equations dealing with vectors. Nonlinear Anal. 72, 4518-4529 (2010)

16. Wu, K, Wu, X: Multiplicity results of periodic solutions for systems of first order delay differential equation. Appl. Math. Comput. 218, 1765-1773 (2011)

17. Wu, K: Multiplicity results of periodic solutions for a class of first order delay differential equations. J. Math. Anal. Appl. $390,427-438(2012)$

18. Wu, K, Wu, X, Zhou, F: Multiplicity results of periodic solutions for a class of second order delay differential equations. Nonlinear Anal. 75, 5836-5844 (2012)

19. $\mathrm{Yu}, J, \mathrm{Xiao}, \mathrm{H}$ : Multiplicity periodic solutions with minimal period 4 of delay differential equations $x^{\prime}(t)=-f(t, x(t-1))$. J. Differ. Equ. 254, 2158-2172 (2013)

20. Yu, JS: A note on periodic solutions of the delay differential equation $x^{\prime}(t)=-f(x(t-1))$. Proc. Am. Math. Soc. 141, 1267-1280 (2013)

21. Zhang, X, Meng, Q: Nontrivial periodic solutions for delay differential systems via Morse theory. Nonlinear Anal. 74, 1960-1968 (2011)

\section{Submit your manuscript to a SpringerOpen ${ }^{\circ}$ journal and benefit from:}

- Convenient online submission

- Rigorous peer review

- Immediate publication on acceptance

- Open access: articles freely available online

- High visibility within the field

- Retaining the copyright to your article 